\title{
Brief versions of the FACIT-fatigue and FAACT subscales for patients with non-small cell lung cancer cachexia
}

\author{
John M. Salsman • Jennifer L. Beaumont • \\ Katy Wortman • Ying Yan • John Friend • David Cella
}

Received: 4 June 2014 / Accepted: 14 October 2014 / Published online: 29 October 2014

(C) The Author(s) 2014. This article is published with open access at Springerlink.com

\begin{abstract}
Purpose Cancer anorexia-cachexia syndrome (CACS) is common in advanced cancer patients and associated with weight loss, fatigue, impaired quality of life (QoL), and poor prognosis. The goal of this project was to identify the most responsive items from two QoL measures in the ROMANA 2 (NCT01387282) phase III global study evaluating anamorelin $\mathrm{HCl}$ in the treatment of non-small cell lung cancer (NSCLC) cachexia: the Functional Assessment of Chronic Illness Therapy-Fatigue (FACIT-F) and the Functional Assessment of Anorexia/Cachexia Therapy (FAACT).

Methods In the ROMANA 2 trial, 477 patients with unresectable stage III or IV NSCLC and cachexia were to be enrolled and randomized (2:1) to receive anamorelin $\mathrm{HCl}$ or placebo once daily for 12 weeks. All 203 patients who reached the week 12 visit at the time of data analysis were included. Co-primary endpoints were change from baseline in lean body mass and handgrip strength. QoL was a secondary outcome with FACIT-F and FAACT questionnaires administered at baseline and at weeks 3, 6, 9, and 12 .

Results Two 4-item scales (fatigue/activity and appetite/eating) from the FACIT-F and FAACT questionnaires, respectively, demonstrated good internal consistency reliability, validity, and responsiveness (also referred to as the Simplified Evaluation of Fatigue (SEF) and Simplified Evaluation of
\end{abstract}

J. M. Salsman $(\bowtie) \cdot$ J. L. Beaumont $\cdot$ K. Wortman $\cdot$ D. Cella Department of Medical Social Sciences, Feinberg School of Medicine at Northwestern University, 633 North St. Clair, 19th Floor, Chicago, IL 60611, USA

e-mail: j-salsman@northwestern.edu

J. M. Salsman • D. Cella

Robert H. Lurie Comprehensive Cancer Center of Northwestern

University, Chicago, IL 60611, USA

Y. Yan · J. Friend

Helsinn Therapeutics (US), INC., Bridgewater, NJ 08807, USA
Appetite (SEA), respectively). The estimated important difference for each scale was 1-2 points.

Conclusions These brief scales provide the psychometric properties necessary to promote future research in NSCLC patients with CACS. Additional work should examine the clinical utility of these scales and their impact on treatment decision-making.

Keywords Measurement $\cdot$ Anorexia $\cdot$ Cachexia $\cdot$ Fatigue $\cdot$ Quality of life

\section{Introduction}

Cancer anorexia-cachexia syndrome (CACS) is a multifactorial condition characterized by an ongoing loss of skeletal muscle mass that leads to progressive functional impairment and cannot be fully reversed through conventional nutritional support [1,2]. CACS affects a majority of patients with advanced cancer and is associated with response to therapy, a reduction in treatment tolerance, duration of survival, and quality of life [3]. Accordingly, weight loss and decreased muscle mass are conditions that warrant close monitoring in patients with solid tumors, including those with lung cancer.

Lung cancer is the leading cause of cancer-related deaths worldwide with an estimated 1.37 million deaths attributed to lung cancer in 2008 [4]. In the USA, $50 \%$ of newly diagnosed patients with non-small cell lung cancer (NSCLC) present with advanced disease and, in spite of significant advances in treatment, their adjusted 1-year survival ranges from 12 to $27 \%$ [5]. In a prospective study of patients with stages III and IV NSCLC, $58 \%$ of patients reported weight loss and when compared to those who did not report weight loss, those who did had significantly lower response rates, shorter progression-free survival, and shorter overall survival [6]. 
In addition to a direct impact on response and survival, maintaining adequate nutrition and minimizing weight loss is a concern for many patients and their families [7]. A number of benefits in addition to survival might be associated with halting the progression of CACS, including enhancing physical ability and improving quality of life [8]. CACS in patients with NSCLC is an important area of study given the prevalence of the disease, the frequency with which it is associated with weight loss, and the prognostic implications of weight loss for patients with advanced NSCLC. Unfortunately, brief, valid, and reliable patient-reported outcomes of cachexia are lacking.

Anamorelin $\mathrm{HCl}$ is an orally active ghrelin receptor agonist with appetite-enhancing and anabolic activity. Based on the data available from phase I and phase II clinical trials, anamorelin $\mathrm{HCl}$ produced an increase in total body weight and lean body mass in patients with advanced cancer, and specifically in patients with NSCLC, in addition to increasing muscle strength and improving quality of life outcomes [9-13]. Anamorelin is currently being evaluated in three phase III studies in patients with NSCLC cachexia, including ROMANA 2 (NCT01387282), a randomized, double-blind, placebo-controlled, multicenter study to evaluate safety and efficacy [14].

The goal of this project was (1) to identify, blind to treatment assignment, the most responsive items from among selfreport measures administered in the ROMANA 2 phase III global study, and (2) to evaluate the validity of the selected subset of questions for the patient population. Identification of a brief subset of items to assess cachexia in the context of NSCLC would thus address an important gap in the research literature and provide a useful assessment tool to facilitate treatment decision-making and enhance patient-centered care.

\section{Methods}

Samples and procedures

Patient recruitment for the ROMANA 2 trial took place in 7 countries at approximately 50 research sites. Written consent was obtained from all participants, and the study protocol was approved by each site's institutional review board. Patients were eligible to participate in the study if they were/had $\geq 18$ years of age, diagnosed with unresectable stage III or IV NSCLC, involuntary weight loss of $\geq 5 \%$ body weight within 6 months prior to screening or a screening body mass index (BMI) $<20 \mathrm{~kg} / \mathrm{m}^{2}$, a body mass index $\leq 30 \mathrm{~kg} / \mathrm{m}^{2}$, an ECOG performance status $\leq 2$, an estimated life expectancy of $>4$ months, adequate hepatic and renal function, and able to understand and comply with the protocol tests and procedures. Patients may have been receiving maintenance chemotherapy, planning to initiate a new chemotherapy and/or radiation therapy regimen (within \pm 14 days of randomization), or may have completed a chemotherapy and/or radiation therapy and/ or have no plan to initiate a new regimen within 12 weeks from randomization (at least 14 days must elapse from the completion of the chemotherapy and/or radiation therapy prior to randomization).

A total of 477 patients were planned to be randomized to receive either anamorelin $\mathrm{HCl}$ or placebo in a 2:1 ratio. Via an interactive voice response system (IVRS), central randomization stratified patients by geographic region (North America vs. rest of world), by chemotherapy and/or radiation therapy status (patients who initiated chemotherapy and/or radiation therapy within \pm 14 days of randomization or patients who were only receiving maintenance chemotherapy vs. patients who had no plan to initiate chemotherapy and/or radiation therapy within 12 weeks from randomization), and by weight loss over the prior 6 months ( $\leq 10 \%$ of body weight vs. $>10 \%$ of body weight).

\section{Study measures}

Demographic information obtained included age, gender, race, ethnicity, and geographic region. Clinical information obtained included Karnofsky performance status (KPS), body weight, BMI, lean body mass (as measured by dual energy Xray absorptiometry), muscle strength (as measured by handgrip strength), and the Hunger Assessment Scale (HAS), developed specifically for this trial. The HAS was comprised of two items: "I have felt hungry," and "My family and friends are pleased with my appetite." The HAS was analogous to the subjective hunger index from the Grand Hunger Scale [15], and participants responded to the items using a $0-4$ Likert scale. Quality of life outcome measures included the Functional Assessment of Cancer Therapy-General (FACTG) [16], Functional Assessment of Chronic Illness TherapyFatigue (FACIT-F) Subscale [17], and the Functional Assessment of Anorexia/Cachexia Therapy (FAACT) [18]. The FACIT-F and FAACT have been previously used and validated in clinical research investigations. The instruments were provided using certified translations (as available) and completed by patients in their native language. All assessments were administered at baseline and then at weeks $3,6,9$, and 12 of the treatment period.

\section{Analytic strategy}

This project followed several steps. First, we analyzed the ROMANA 2 phase III trial data on a blinded basis to identify the most responsive items using a preliminary database that included all 203 patients who had reached the week 12 visit at the time of data analysis. Second, the most responsive items were reviewed by the research team at Northwestern University and Helsinn to identify a "best" list of items to 
analyze further. Third, the "best" items were validated and analyzed to identify the size of a meaningful difference and provide clinical interpretation.

Compliance We calculated compliance rates for each question on the FACIT-F and FAACT as the proportion of patients who completed the question at each assessment from those who completed any question in that assessment.

Identification of most responsive items We conducted a series of analyses to aid in the selection of a brief subset of the most responsive items. First, change scores were calculated for all FACIT-F and FAACT items as the week 12 score minus the baseline score. Then, we formed groups of patients based on whether their KPS improved, worsened, or stayed the same. Analysis of variance (ANOVA) was used to compare item change scores between these groups. A similar set of item change scores was used to compare patients classified into similar groups (improved, worsened, or unchanged) as determined by change in lean body mass, handgrip strength, and body weight.

Effect sizes to aid in the interpretation of the magnitude of the change were calculated. Items were flagged as "responsive" if they met the following criteria: (1) the effect size for at least one of the change groups was $>0.30$ in magnitude and in the expected direction, (2) the effect size in the other change group was in the expected direction, and (3) the effect size for the unchanged group fell between. Pearson correlation coefficients were calculated between the item change scores and the changes in KPS, lean body mass, handgrip strength, body weight, and the Hunger Assessment Scale items. Items were considered responsive if the correlation coefficient was greater than 0.25 . These criteria were defined based on standard guidelines for interpreting effect sizes and correlations while also targeting the identification of a brief number of "most responsive" items.

Internal consistency reliability Pearson correlation coefficients were calculated among all pairs of responsive items at each visit. Cronbach's coefficient alpha was calculated to evaluate the internal consistency reliability of that set of items. We also examined these item-total correlations to identify items with poor correlations with the total.

Known-groups validity We conducted cross-sectional analyses of scores focused on differentiating definable ("known") groups according to appropriate clinical anchor variables. Groups were formed according to KPS, the questions of the Hunger Assessment Scale, lean body mass, and handgrip strength. We used ANOVA to compare mean scores between these groups. We expected that patients with worse performance/hunger status would have worse mean summary scores. Effect sizes (mean difference/pooled standard deviation) were calculated for group comparisons to provide an indication of the magnitude of these group differences.

Convergent validity We hypothesized that the questionnaire summary scores would be associated with fatigue, as measured by the FACIT-F scale, anorexia/cachexia, as measured by the FAACT, and general quality of life/functioning, as measured by the FACT-G. We further anticipated that scores would be associated with clinical variables and the items of the Hunger Assessment Scale. The association between the summary scores and these other measures was evaluated using Pearson correlations at each assessment. Moderate correlations in the range of 0.3 to 0.7 were expected.

Responsiveness As in the assessment of convergent validity, we hypothesized that changes in the summary scores would be moderately associated with changes in the FACIT-F, FACT-G, FAACT, lean body mass, handgrip strength, body weight, and Hunger Assessment Scale. To evaluate this, first change scores were calculated for all instruments by subtracting the baseline score. Pearson correlation coefficients were then calculated between the brief scale change scores and the FACIT-F, FACT-G, FAACT, lean body mass, handgrip strength, body weight, and Hunger Assessment Scale change scores.

We formed groups of patients based on whether their performance status improved, worsened, or stayed the same. ANOVA was used to compare questionnaire change scores between these groups. A similar comparison of change scores was used to compare patients between change groups defined by lean body mass change, handgrip strength change, and body weight change. Effect sizes were calculated.

Important differences and clinical interpretation Distributionand anchor-based methods were used to identify important differences for the new scale scores. Based upon other work $[19,20]$, distribution-based methods include $1 / 3$ standard deviation (SD), 1/2 SD, and one standard error of measurement (SEM). Anchor-based methods [21] utilized crosssectional and longitudinal data.

The randomized treatment arms were collapsed for all analyses described above and data remained blinded. There was no imputation of missing data and no adjustment for multiple comparisons. Analyses were conducted with SAS v9.3 (SAS Institute, Cary, NC).

\section{Results}

Descriptive characteristics of sample

A preliminary database lock was used for this study, selecting all patients who had reached the 12-week follow-up time 
when the data were pulled (February 2013). A total of 383 enrolled patients were included at the time of data analysis. Baseline demographic and clinical characteristics of the sample are presented in Table 1. The mean age was 63.3 years $(\mathrm{SD}=8.7$, range $=33$ to 88$)$.

\section{Compliance}

The number of patients remaining on study at each assessment was $325,274,233$, and 203, for weeks $3,6,9$, and 12 , respectively. Item level compliance for the FACIT-F and FAACT was greater than $95 \%$ for all items except for optional item GS7 ("I am satisfied with my sex life") which had compliance levels of $34-41 \%$.

\section{Confirmation of most responsive items}

Table 2 summarizes the results of the group and correlation analyses. The items were sorted according to the number of

Table 1 Baseline sample characteristics $(N=383)$

\begin{tabular}{lll}
\hline Demographic characteristics & $N$ & $\%$ \\
\hline Gender & & \\
$\quad$ Female & 104 & $27 \%$ \\
$\quad$ Male & 279 & $73 \%$ \\
Race/ethnicity & & \\
$\quad$ Asian & 1 & $0 \%$ \\
$\quad$ Black or African American & 2 & $1 \%$ \\
$\quad$ Hispanic or Latino & 1 & $0 \%$ \\
$\quad$ Other & 2 & $1 \%$ \\
$\quad$ White & 378 & $99 \%$ \\
Country & & \\
$\quad$ Poland & 176 & $24 \%$ \\
$\quad$ Russia & 92 & $20 \%$ \\
$\quad$ Hungary & 75 & $5 \%$ \\
$\quad$ Australia & 19 & $3 \%$ \\
$\quad$ United States of America & 11 & $2 \%$ \\
Israel & 9 & $0 \%$ \\
$\quad$ United Kingdom & 1 & Range \\
Clinical characteristics & Mean (SD) & $2-48$ \\
Karnofsky performance status & $79.3(9.8)$ & $50-100$ \\
Body mass index & $22.2(3.7)$ & $14-30$ \\
Body weight, kg & $63.0(13.0)$ & $34-98$ \\
Nondominant handgrip strength & $28.6(11.4)$ & $5-58$ \\
Lean body mass, kg & $43.9(8.1)$ & \\
Patient-reported outcome scores & Mean (SD) \\
FAACT subscale & $27.7(8.8)$ & \\
FACIT-fatigue subscale & $27.5(10.8)$ & \\
FACT-G & $64.3(15.9)$ & \\
\hline
\end{tabular}

variables to which they were responsive in these two analyses. The top eight items were selected as the subset of the most responsive items: ACT1-The amount I eat is sufficient to meet my needs, ACT9-Family/friends are pressuring me to eat, ACT10-When I eat I seem to get full quickly, C6-I have a good appetite, AN12-I am too tired to eat, GP7-I am forced to spend time in bed, HI7-I feel fatigued, and HI12-I feel weak all over. These eight items fell into two distinct categories with the first four measuring appetite/eating and the last four measuring fatigue/activity. Therefore, two separate scores were created by summing the responses to the four items in each scale. The validity of these two 4item scale scores was assessed in the remainder of this report.

Internal consistency reliability

Fatigue/activity items were highly intercorrelated with interitem correlations of 0.45 or greater at each time. Inter-item correlations among appetite/eating items were generally greater than 0.20 with the exception of ACT9 (family/friends pressuring to eat) with ACT1 (amount eat sufficient to meet needs). Cronbach's coefficient alpha for the fatigue/activity scale was $0.83,0.84,0.84,0.85$, and 0.87 at baseline and weeks $3,6,9$, and 12 , respectively. All item-total correlations were at least 0.55 . Cronbach's coefficient alpha for the appetite/eating scale was $0.71,0.73,0.71,0.73$, and 0.76 at baseline and weeks 3, 6, 9, and 12, respectively. All item-total correlations were greater than 0.35 .

Scale scores

The brief 4-item fatigue/activity and appetite/eating scores (also referred to as the Simplified Evaluation of Appetite (SEA) and Simplified Evaluation of Fatigue (SEF), respectively) were calculated according to standard FACIT measurement system guidelines. Specifically, negatively worded items were reverse scored and then all item scores were summed to create total scores such that higher scores indicated better quality of life or lower symptom levels. Descriptive statistics for these calculated scores are presented in Table 3.

Known-groups validity

At baseline, fatigue/activity scores differed between KPS groups with effect sizes for differences between adjacent groups of $0.34(p=0.001)$. A similar relationship was observed at weeks 6 and 12, with slightly larger effect sizes of 0.38 to $0.53(p<0.001)$. Although not previously hypothesized, fatigue/activity scores also differed between groups defined by nondominant handgrip strength and the Hunger 
Table 2 Summary of item responsiveness

Change from baseline to

week 12 in..
KPS

BMI Lean Body

Mass
Handgrip

strength

\section{Body}

weigh
"I have felt

hungry"
"My family and Total friends are pleased count with my appetite"
ACT10: When I eat I seem to get full quickly

ACT1: Amount I eat sufficient to meet my needs

ACT9: Family/friends are pressuring me to eat

AN12: I am too tired to eat

C6: I have a good appetite

GP7: I am forced to spend time in bed

HI12: I feel weak all over

HI7: I feel fatigued

ACT6: Interest in food drops when I try to eat

AN2: I feel tired

AN4: Trouble finishing things because I'm tired

GF2: My work is fulfilling

GP6: I feel ill

ACT3: Most food tastes unpleasant to me

ACT4: I am concerned about how thin I look

AN1: I feel listless ('washed out')

AN3: Trouble starting things because I'm tired

AN5: I have energy

AN8: I need to sleep during the day

GE1: I feel sad

$\mathrm{O} 2$ : I have been vomiting

ACT13: My general health is improving

ACT7: Difficulty eating rich/heavy foods

AN7: I am able to do my usual activities

GF1: I am able to work (include work at home)

GP1: I have a lack of energy

GP5: I am bothered by side effects of treatment

ACT2: I am worried about my weight

AN16: I limit my social activity because too tired

GE6: I worry that my condition will get worse

GF3: I am able to enjoy life

GF6: I'm enjoying things I usually do for fun

GP2: I have nausea

GP3: I have trouble meeting needs of my family

GS3: I get support from my friends

GS7: I am satisfied with my sex life

ACT11: I have pain in my stomach area

AN14: I need help doing my usual activities

AN15: Frustrated by being too tired to do things

GE2: Satisfied w/how I'm coping w/my illness

GE3: Losing hope in fight against my illness

GE4: I feel nervous

GE5: I worry about dying

GF4: I have accepted my illness

GF5: I am sleeping well

GF7: Content w/quality of my life right now

GP4: I have pain

GS1: I feel close to my friends

\begin{tabular}{|c|c|c|c|c|c|c|}
\hline \multirow[t]{2}{*}{ O } & XO & & $\mathrm{O}$ & XO & $X$ & $X$ \\
\hline & XO & $\mathrm{O}$ & & XO & $X$ & $\mathrm{X}$ \\
\hline $\mathrm{O}$ & XO & XO & & $\mathrm{O}$ & & $\mathrm{X}$ \\
\hline \multirow[t]{2}{*}{ O } & $\mathrm{O}$ & $\mathrm{O}$ & & $\mathrm{O}$ & $\mathrm{X}$ & $\mathrm{X}$ \\
\hline & XO & & & XO & $X$ & $X$ \\
\hline XO & $\mathrm{O}$ & $\mathrm{O}$ & & & $\mathrm{X}$ & $\mathrm{X}$ \\
\hline $\mathrm{O}$ & $\mathrm{O}$ & $\mathrm{O}$ & & $\mathrm{O}$ & & $X$ \\
\hline \multirow[t]{2}{*}{$\mathrm{O}$} & $\mathrm{O}$ & $\mathrm{O}$ & & $\mathrm{O}$ & & $X$ \\
\hline & $\mathrm{O}$ & & & $\mathrm{O}$ & $X$ & $\mathrm{X}$ \\
\hline \multirow[t]{2}{*}{$\mathrm{O}$} & $\mathrm{O}$ & & & $\mathrm{O}$ & & X \\
\hline & $\mathrm{O}$ & $\mathrm{O}$ & & $\mathrm{O}$ & & $X$ \\
\hline XO & $\mathrm{O}$ & & & & & $\mathrm{X}$ \\
\hline \multirow[t]{3}{*}{$\mathrm{O}$} & O & $\mathrm{O}$ & & & & $\mathrm{X}$ \\
\hline & $\mathrm{O}$ & & & $\mathrm{O}$ & & $X$ \\
\hline & & XO & & & & $X$ \\
\hline \multirow[t]{3}{*}{$\mathrm{O}$} & & & & & $X$ & $\mathrm{X}$ \\
\hline & $\mathrm{O}$ & $\mathrm{O}$ & & & & $X$ \\
\hline & & $\mathrm{O}$ & & $\mathrm{O}$ & & $X$ \\
\hline XO & $\mathrm{O}$ & & & & & \\
\hline \multirow[t]{4}{*}{$\mathrm{O}$} & & $\mathrm{O}$ & & & & $\mathrm{X}$ \\
\hline & $\mathrm{O}$ & & & & $X$ & $X$ \\
\hline & $X$ & & & & & $\mathrm{X}$ \\
\hline & & & & $\mathrm{O}$ & & $X$ \\
\hline $\mathrm{O}$ & & & & & & $X$ \\
\hline \multirow[t]{5}{*}{$\mathrm{O}$} & $\mathrm{O}$ & & & & & \\
\hline & & $\mathrm{O}$ & & $\mathrm{O}$ & & \\
\hline & $\mathrm{O}$ & & & & & $\mathrm{X}$ \\
\hline & & & & & & $X$ \\
\hline & & & & & & $\mathrm{X}$ \\
\hline
\end{tabular}

$\mathrm{O}$

$\mathrm{X}$

8

7

7

6

3

3

3

3

2

2

2

2

2

2

1

1

1

1

O

O 
Table 2 (continued)

\begin{tabular}{|c|c|c|c|c|c|c|c|c|}
\hline $\begin{array}{l}\text { Change from baseline to } \\
\text { week } 12 \text { in... }\end{array}$ & KPS & BMI & $\begin{array}{l}\text { Lean Body } \\
\text { Mass }\end{array}$ & $\begin{array}{l}\text { Handgrip } \\
\text { strength }\end{array}$ & $\begin{array}{l}\text { Body } \\
\text { weight }\end{array}$ & $\begin{array}{l}\text { "I have felt } \\
\text { hungry" }\end{array}$ & $\begin{array}{l}\text { "My family and } \\
\text { friends are pleased } \\
\text { with my appetite" }\end{array}$ & $\begin{array}{l}\text { Total } \\
\text { count }\end{array}$ \\
\hline
\end{tabular}

GS2: I get emotional support from my family

GS4: My family has accepted my illness

GS5: Satisfied with family communication about my illness

$X$ responsive in correlation analysis, $O$ responsive in grouped analysis

Assessment Scale item "My family and friends are pleased with my appetite" at all assessments $(p<0.005)$.

Baseline appetite/eating scores differed significantly between "I have felt hungry" groups $(p<0.001)$ but effect sizes for differences between adjacent groups were small to moderate $(0.13$ to 0.56$)$. For "My family and friends are pleased with my appetite" groups, the effect sizes for differences between adjacent group were large ( 0.66 to $0.84, p<0.001)$. Again, similar relationships were observed at weeks 6 and 12 , with slightly larger effect sizes (0.37 to $1.31, p<0.001)$. Although not previously hypothesized, appetite/eating scores also differed between groups defined by nondominant handgrip strength and KPS at all assessments $(p<0.004)$.

\section{Convergent validity}

Pearson correlation coefficients at each assessment between the brief scale scores and the existing measures are presented in Table 4. As hypothesized, the fatigue/activity and appetite/eating scores were associated with fatigue, as measured by the FACIT-F scale, anorexia/cachexia, as measured by the FAACT, and general quality of life/functioning, as measured by the FACT-G. Fatigue/ activity scores were also moderately correlated with responses to the HAS item "My family and friends are pleased with my appetite." Appetite/eating scores were more strongly correlated with that item and moderately correlated with "I have felt

Table 3 Descriptive statistics for fatigue/activity and appetite/eating scores

\begin{tabular}{llllll}
\hline & Baseline & Week 3 & Week 6 & Week 9 & Week 12 \\
\hline Fatigue/activity & & & & & \\
$N$ & 378 & 314 & 273 & 227 & 196 \\
Mean (SD) & $9.1(3.7)$ & $9.4(3.9)$ & $9.8(3.8)$ & $10.0(3.8)$ & $9.7(3.8)$ \\
Range & $0-16$ & $0-16$ & $0-16$ & $1-16$ & $1-16$ \\
Appetite/eating & & & & & \\
$N$ & 378 & 312 & 273 & 226 & 196 \\
Mean (SD) & $8.0(3.8)$ & $9.5(3.8)$ & $9.7(3.5)$ & $9.7(3.6)$ & $9.8(3.6)$ \\
Range & $0-16$ & $0-16$ & $1-16$ & $1-16$ & $0-16$ \\
\hline
\end{tabular}

hungry." Correlations with KPS and nondominant handgrip strength were small to moderate for both scales.

\section{Responsiveness}

Pearson correlation coefficients between the brief scale change scores and the FACIT-F, FACT-G, FAACT, lean body mass, handgrip strength, body weight, and Hunger Assessment Scale change scores are presented in Table 5. As hypothesized, changes in fatigue/activity and appetite/eating scores were associated with changes in fatigue, as measured by the FACIT-F scale, anorexia/cachexia, as measured by the FAACT, and general quality of life/functioning, as measured by the FACT-G. Fatigue/activity change scores were also moderately correlated with changes in responses to the item "My family and friends are pleased with my appetite." Appetite/eating change scores were more strongly correlated with changes in that item and moderately correlated with changes in "I have felt hungry" and body weight. Correlations with changes in KPS and nondominant handgrip strength were small for both scales.

Important differences and clinical interpretation

The distribution-based estimates are summarized in Table 6. Of the cross-sectional anchor-based analyses, many effect sizes for comparison of KPS and "My family and friends are pleased with my appetite" groups met the criteria for consideration and are listed in Table 6. For the longitudinal analyses, however, only the body weight change group comparisons met the criteria and only for the appetite/eating score. The estimated (minimally) important difference for the fatigue/activity scale was 1.3 to 1.9 points; for the appetite/eating scale, it was 1.2 to 2.0 points; that is, roughly $1-2$ points for both scales.

\section{Discussion}

This study identified eight of the most responsive items from the FACIT-F and FAACT scales for use in patients undergoing 
Table 4 Correlations between brief scale scores and existing measures (convergent validity)

\begin{tabular}{|c|c|c|c|c|c|}
\hline Variable & Baseline & Week 3 & Week 6 & Week 9 & Week 12 \\
\hline \multicolumn{6}{|l|}{ Correlation with fatigue/activity scores } \\
\hline Nondominant handgrip strength & $0.26^{* *}$ & NA & $0.27 * *$ & NA & $0.28^{* *}$ \\
\hline FAACT & $0.68 * *$ & $0.77 * *$ & $0.83 * *$ & $0.76^{* *}$ & $0.76^{* *}$ \\
\hline FACIT-fatigue scale & $0.91 * *$ & $0.92 * *$ & $0.93 * *$ & $0.93 * *$ & $0.91 * *$ \\
\hline FACT-G physical well-being & $0.74 * *$ & $0.83 * *$ & $0.83 * *$ & $0.81 * *$ & $0.78^{* *}$ \\
\hline FACT-G emotional well-being & $0.47 * *$ & $0.62 * *$ & $0.65 * *$ & $0.52 * *$ & $0.59 * *$ \\
\hline FACT-G social/family well-being & 0.04 & 0.09 & $0.20 * *$ & $0.19^{*}$ & 0.10 \\
\hline FACT-G functional well-being & $0.41 * *$ & $0.42 * *$ & $0.51 * *$ & $0.50 * *$ & $0.52 * *$ \\
\hline FACT-G total & $0.59 * *$ & $0.71 * *$ & $0.77 * *$ & $0.69 * *$ & $0.68 * *$ \\
\hline Karnofsky performance status & $0.23 * *$ & $0.22 * *$ & $0.31 * *$ & $0.20^{*}$ & $0.37 * *$ \\
\hline Lean body mass & $0.19 * *$ & NA & $0.13 *$ & NA & $0.15^{*}$ \\
\hline Body weight & $0.18^{* *}$ & $0.16^{*}$ & 0.11 & 0.12 & $0.16^{*}$ \\
\hline I have felt hungry & $0.12 *$ & 0.06 & 0.10 & 0.09 & $0.19 *$ \\
\hline My family and friends are pleased with my appetite & $0.38^{* *}$ & $0.29 * *$ & $0.33^{* *}$ & $0.37 * *$ & $0.46^{* *}$ \\
\hline \multicolumn{6}{|l|}{ Correlation with appetite/eating scores } \\
\hline Nondominant handgrip strength & $0.23 * *$ & NA & $0.23 * *$ & NA & $0.27 * *$ \\
\hline FAACT & $0.68^{* *}$ & $0.69^{* *}$ & $0.74 * *$ & $0.75^{* *}$ & $0.77 * *$ \\
\hline FACIT-Fatigue scale & $0.56^{* *}$ & $0.49 * *$ & $0.60 * *$ & $0.58 * *$ & $0.66^{* *}$ \\
\hline FACT-G physical well-being & $0.45^{* *}$ & $0.49^{* *}$ & $0.54 * *$ & $0.57 * *$ & $0.64 * *$ \\
\hline FACT-G emotional well-being & $0.29 * *$ & $0.38^{* *}$ & $0.44 * *$ & $0.41^{* *}$ & $0.47 * *$ \\
\hline FACT-G social/family well-being & 0.09 & 0.07 & 0.12 & $0.18^{*}$ & 0.07 \\
\hline FACT-G functional well-being & $0.45^{* *}$ & $0.44 * *$ & $0.44 * *$ & $0.46^{* *}$ & $0.53 * *$ \\
\hline FACT-G total & $0.46^{* *}$ & $0.49 * *$ & $0.54 * *$ & $0.55^{* *}$ & $0.59 * *$ \\
\hline Karnofsky performance status & $0.20 * *$ & $0.26^{* *}$ & $0.31 * *$ & $0.30^{* *}$ & $0.27 * *$ \\
\hline Lean body mass & $0.18 * *$ & NA & $0.17 *$ & NA & $0.19 *$ \\
\hline Body weight & $0.25 * *$ & $0.22 * *$ & $0.21 * *$ & $0.32 * *$ & $0.19 *$ \\
\hline I have felt hungry & $0.27 * *$ & $0.37 * *$ & $0.29 * *$ & $0.27 * *$ & $0.35^{* *}$ \\
\hline My family and friends are pleased with my appetite & $0.70 * *$ & $0.68^{* *}$ & $0.65^{* *}$ & $0.67 * *$ & $0.71 * *$ \\
\hline
\end{tabular}

$N A$ not applicable as handgrip strength and lean body mass were only assessed at baseline, week 6 and week 12

$* p<0.05 ; * * p<0.001$

therapy for cancer-related anorexia/cachexia. These eight items were scored as two scales of four items each: fatigue/activity and appetite/eating. Scores differed between groups defined by performance status, nondominant handgrip strength, lean body mass, and items assessing self-perceptions of hunger and others' satisfaction with patient's appetite. As expected, fatigue/activity and appetite/eating scores were associated with fatigue, anorexia/cachexia, and general quality of life.

The fatigue/activity and appetite/eating scales demonstrated strong psychometric properties. These two scales had good internal consistency reliability across multiple assessments and when examining change from baseline. In addition, changes in fatigue/activity and appetite/eating scores were not merely limited to associations with changes in fatigue (FACIT-F) and appetite (Hunger Assessment Scale), respectively, but were also associated with changes in anorexia/cachexia, general quality of life, and body weight with those gaining (or losing) the most weight reporting small but significant improvements (or declines) in scores. Similar associations were seen with change in lean body mass. Collectively, these findings underscore the potential utility of these brief subscales for assessing and better understanding cachexia in patients with advanced NSCLC.

Importantly, the brief versions of the FACIT-F and FAACT subscales compared quite favorably to their longer counterparts. Within this current sample, the correlations between the fatigue/activity and the FACIT-F and between the appetite/ eating and the FAACT were strong across all assessments, $r=$ 0.91 to 0.93 and $r=0.68$ to 0.77 (Table 4 ). When compared to the validation samples [18, 22], internal consistency for the fatigue/activity scale had slightly lower internal consistency (range $0.83-0.87$ vs. $0.93-0.95$ ), but the appetite/eating scale 
Table 5 Correlations between brief scale change scores and existing measures' change scores (responsiveness)

\begin{tabular}{|c|c|c|c|c|}
\hline Change from baseline in... & Week 3 & Week 6 & Week 9 & Week 12 \\
\hline \multicolumn{5}{|l|}{ Correlation with fatigue/activity change scores } \\
\hline Nondominant handgrip strength & NA & $0.19^{*}$ & NA & 0.08 \\
\hline FAACT & $0.60 * *$ & $0.62 * *$ & $0.66^{* *}$ & $0.71 * *$ \\
\hline FACIT-fatigue scale & $0.84 * *$ & $0.84 * *$ & $0.82 * *$ & $0.86^{* *}$ \\
\hline FACT-G physical well-being & $0.68 * *$ & $0.70 * *$ & $0.67 * *$ & $0.69 * *$ \\
\hline FACT-G emotional well-being & $0.47 * *$ & $0.41 * *$ & $0.37 * *$ & $0.46^{* *}$ \\
\hline FACT-G social/family well-being & -0.05 & $-0.17 *$ & 0.12 & 0.11 \\
\hline FACT-G functional well-being & $0.30 * *$ & $0.30 * *$ & $0.36^{* *}$ & $0.40^{* *}$ \\
\hline FACT-G total & $0.55^{* *}$ & $0.52 * *$ & $0.58^{* *}$ & $0.64 * *$ \\
\hline Karnofsky performance status & $0.13 *$ & $0.17 *$ & $0.20^{*}$ & $0.23^{*}$ \\
\hline Lean body mass & NA & $0.18^{*}$ & NA & $0.18^{*}$ \\
\hline Body weight & $0.19 * *$ & $0.22 * *$ & $0.18^{*}$ & $0.28 * *$ \\
\hline I have felt hungry & 0.11 & $0.15 *$ & $0.23 * *$ & $0.28 * *$ \\
\hline My family and friends are pleased with my appetite & $0.29 * *$ & $0.28 * *$ & $0.39 * *$ & $0.43^{* *}$ \\
\hline \multicolumn{5}{|l|}{ Correlation with appetite/eating change scores } \\
\hline Nondominant handgrip strength & NA & 0.11 & NA & 0.13 \\
\hline FAACT & $0.62 * *$ & $0.69 * *$ & $0.70^{* *}$ & $0.75^{* *}$ \\
\hline FACIT-fatigue scale & $0.38^{* *}$ & $0.44 * *$ & $0.40 * *$ & $0.50 * *$ \\
\hline FACT-G physical well-being & $0.37 * *$ & $0.43 * *$ & $0.49^{* *}$ & $0.56^{* *}$ \\
\hline FACT-G emotional well-being & $0.29 * *$ & $0.25 * *$ & $0.19^{*}$ & $0.33^{* *}$ \\
\hline FACT-G social/family well-being & -0.03 & -0.06 & $0.18^{*}$ & 0.11 \\
\hline FACT-G functional well-being & $0.21 * *$ & $0.24 * *$ & $0.27 * *$ & $0.30 * *$ \\
\hline FACT-G total & $0.32 * *$ & $0.36^{* *}$ & $0.44 * *$ & $0.50 * *$ \\
\hline Karnofsky performance status & $0.18^{*}$ & $0.14 *$ & $0.25^{* *}$ & $0.18^{*}$ \\
\hline Lean body mass & NA & $0.27 * *$ & NA & $0.25^{* *}$ \\
\hline Body weight & $0.30 * *$ & $0.37 * *$ & $0.36^{* *}$ & $0.40^{* *}$ \\
\hline I have felt hungry & $0.27 * *$ & $0.35 * *$ & $0.31^{* *}$ & $0.38^{* *}$ \\
\hline My family and friends are pleased with my appetite & $0.54 * *$ & $0.56^{* *}$ & $0.56^{* *}$ & $0.65^{* *}$ \\
\hline
\end{tabular}

$N A$ not applicable as handgrip strength and lean body mass were only assessed at baseline, week 6 and week 12 $* p<0.05 ; * * p<0.001$

was equivalent (range $0.71-0.76$ vs. 0.76 ). For known-groups validity, the fatigue/activity scale had a significant relationship to performance status scores in the validation sample as well as this current sample (both $p<0.05$ ). The responsiveness of the appetite/eating scale was also quite similar when examining the relationship to performance status in the validation sample compared to the current sample (both $p<0.05$ ).

This study has some limitations. First, the predominantly white, non-Hispanic, male sample may limit the generalizability of the findings. Second, although this study intentionally focused on patients with advanced NSCLC only, cachexia is a significant concern among patients with other types of cancer as well. These brief scales should be tested in other relevant clinical populations. Third, in the process of validating the brief scales, some of our outcomes included redundant item content. We retained overlapping items and reported the findings because they provided the most accurate portrayal of association between original and revised (abbreviated) scales. This was important when evaluating the construct validity of our new measures with well-established and/or face-valid measures.

In summary, this multi-site, longitudinal study included a wide range of important clinical and patient-reported outcomes to identify and validate a brief measure of CACS in patients with advanced stage NSCLC. The results indicated that the fatigue/activity and appetite/eating scales derived from the FACIT-F and FAACT were reliable, valid, and responsive measures. The estimated important difference for each scale was approximately 1 to 2 points, suggesting guidelines for evaluation of clinically significant levels of change and highlighting the utility 
Table 6 Distribution-based and anchor-based estimates of important differences

\begin{tabular}{|c|c|c|c|c|c|}
\hline & & Fatigue/activity & & Appetite/eating & \\
\hline \multirow[t]{5}{*}{$1 / 3 \mathrm{SD}$} & Baseline & 1.2 & & 1.3 & \\
\hline & Week 3 & 1.3 & & 1.3 & \\
\hline & Week 6 & 1.3 & & 1.2 & \\
\hline & Week 9 & 1.3 & & 1.2 & \\
\hline & Week 12 & 1.3 & & 1.2 & \\
\hline \multirow[t]{5}{*}{$1 / 2 \mathrm{SD}$} & Baseline & 1.8 & & 1.9 & \\
\hline & Week 3 & 2.0 & & 1.9 & \\
\hline & Week 6 & 1.9 & & 1.8 & \\
\hline & Week 9 & 1.9 & & 1.8 & \\
\hline & Week 12 & 1.9 & & 1.8 & \\
\hline \multirow[t]{5}{*}{ SEM } & Baseline & 1.5 & & 2.0 & \\
\hline & Week 3 & 1.6 & & 2.0 & \\
\hline & Week 6 & 1.5 & & 1.9 & \\
\hline & Week 9 & 1.5 & & 1.8 & \\
\hline & Week 12 & 1.4 & & 1.8 & \\
\hline \multirow[t]{15}{*}{ Cross-sectional anchors } & \multirow{3}{*}{$\begin{array}{l}\text { Baseline-My friends and family are pleased } \\
\text { with my appetite }\end{array}$} & Not at all vs a little bit & 1.42 & A little bit vs somewhat & 2.15 \\
\hline & & A little bit vs somewhat & 1.35 & Somewhat vs quite a bit & 1.97 \\
\hline & & Somewhat vs quite a bit & 1.20 & Quite a bit vs very much & 2.22 \\
\hline & \multirow[t]{2}{*}{ Week 6-Performance status } & $<70$ vs $70-80$ & 1.61 & $<70$ vs $70-80$ & 1.42 \\
\hline & & $70-80$ vs $90-100$ & 1.42 & $70-80$ vs $90-100$ & 1.55 \\
\hline & \multirow{2}{*}{$\begin{array}{l}\text { Week 6-My friends and family are pleased } \\
\text { with my appetite }\end{array}$} & A little bit vs somewhat & 1.85 & Not at all vs a little bit & 1.16 \\
\hline & & Somewhat vs quite a bit & 1.73 & Quite a bit vs very much & 1.06 \\
\hline & \multirow[t]{2}{*}{ Week 12-Performance status } & $<70$ vs $70-80$ & 1.88 & & \\
\hline & & $70-80$ vs $90-100$ & 1.92 & & \\
\hline & \multirow[t]{2}{*}{ Week $12-$ I have felt hungry } & & & A little bit vs somewhat & 1.94 \\
\hline & & & & Somewhat vs quite a bit & 0.85 \\
\hline & \multirow{4}{*}{$\begin{array}{l}\text { Week } 12-\text { My friends and family are pleased } \\
\text { with my appetite }\end{array}$} & Not at all vs a little bit & 1.24 & Not at all vs a little bit & 2.13 \\
\hline & & A little bit vs somewhat & 2.43 & Somewhat vs quite a bit & 1.14 \\
\hline & & Somewhat vs quite a bit & 0.81 & & \\
\hline & & Quite a bit vs very much & 1.48 & & \\
\hline \multirow[t]{6}{*}{ Longitudinal anchors } & \multirow[t]{2}{*}{ Week 6-Change in body weight } & & & Decreased $3 \mathrm{~kg}$ or more & 0.69 \\
\hline & & & & Increased $1-2.99 \mathrm{~kg}$ & 1.47 \\
\hline & \multirow[t]{2}{*}{ Week 9-Change in body weight } & & & Decreased $3 \mathrm{~kg}$ or more & 2.33 \\
\hline & & & & Increased $1-2.99 \mathrm{~kg}$ & 1.35 \\
\hline & \multirow[t]{2}{*}{ Week 12-Change in body weight } & & & Decreased $3 \mathrm{~kg}$ or more & 2.27 \\
\hline & & & & Increased 1-2.99 kg & 1.21 \\
\hline Median (IQR) & & $1.5(1.3-1.9)$ & & $1.8(1.2-2.0)$ & \\
\hline
\end{tabular}

For anchor-based analyses, only group comparisons that met all three criteria are presented: (1) correlation $\geq 0.3$, (2) group $n \geq 10$, and (3) effect size between 0.2 and 0.8

$I Q R$ interquartile range

of these assessment tools in clinical trials. As mentioned above, future work should examine the generalizability of these findings to patients with other solid tumors who are prone to CACS. In addition, clinicians and researchers alike may benefit from implementing this tool in clinical practice settings to examine ways in which it may facilitate treatment decision-making, improve patient-centered care, and enhance quality of life outcomes for patients suffering from cancer-related cachexia.

Conflict of interest Helsinn Therapeutics (US) Inc. paid Northwestern University for the scientific and consultative expertise of John M. Salsman, Jennifer L. Beaumont, Katy Wortman, and David Cella in connection with the development of this manuscript. Ying Yan and 
John Friend are employees of Helsinn Therapeutics. The authors have no additional financial disclosures related to this manuscript.

Open Access This article is distributed under the terms of the Creative Commons Attribution Noncommercial License which permits any noncommercial use, distribution, and reproduction in any medium, provided the original author(s) and the source are credited.

\section{References}

1. Fearon K, Strasser F, Anker SD, Bosaeus I, Bruera E, Fainsinger RL, Jatoi A, Loprinzi C, MacDonald N, Mantovani G, Davis M, Muscaritoli M, Ottery F, Radbruch L, Ravasco P, Walsh D, Wilcock A, Kaasa S, Baracos VE (2011) Definition and classification of cancer cachexia: an international consensus. Lancet Oncol 12: 489-495

2. Kern K, Norton J (1988) Cancer cachexia. JPEN J Parenter Enteral Nutr 12:286-298

3. Johns N, Stephens NA, Fearon KCH (2013) Muscle wasting in cancer. Int J Biochem Cell Biol 45:2215-2229

4. World Health Organization (2013) Cancer - Fact sheet No. 297. In: Editor (ed)^(eds) Book Cancer - Fact sheet No. 297, City

5. Davidoff AJ, Tang M, Seal B, Edelman MJ (2010) Chemotherapy and survival benefit in elderly patients with advanced non-small cell lung cancer. J Clin Oncol 28:2191-2197

6. Ross PJ, Ashley S, Norton A, Priest K, Waters JS, Eisen T, Smith IE, O'Brien MER (2004) Do patients with weight loss have a worse outcome when undergoing chemotherapy for lung cancers? $\mathrm{Br} \mathrm{J}$ Cancer 90:1905-1911

7. Del Ferraro C, Grant M, Koczywas M, Dorr-Uyemura LA (2012) Management of anorexia-cachexia in late-stage lung cancer patients. J Hosp Palliat Nurs 14:397-402

8. Wallengren O, Lundholm K, Bosaeus I (2013) Diagnostic criteria of cancer cachexia: relation to quality of life, exercise capacity and survival in unselected palliative care patients. Support Care Cancer 21:1569-1577

9. Currow DC, Abernethy AP (2014) Anamorelin hydrochloride in the treatment of cancer anorexia-cachexia syndrome Future Oncol: $1-14$

10. Garcia J, Boccia RV, Graham C, Kumor K, Polvino W (2007) A phase II randomized, placebo-controlled, double-blind study of the efficacy and safety of RC-1291 (RC) for the treatment of cancer cachexia. J Clin Oncol 18:9133

11. Garcia J, Friend J, Allen S (2013) Therapeutic potential of anamorelin, a novel, oral ghrelin mimetic, in patients with cancerrelated cachexia: a multicenter, randomized, double-blind, crossover, pilot study. Support Care Cancer 21:129-137
12. Temel J, Bondarde S, Jain M, Allen S, Mann W (2013) Efficacy and safety of anamorelin $\mathrm{HCl}$ in NSCLC patients: results from a randomized, double-blind, placebo-controlled, multicenter phase II study. European Cancer Congress. Amsterdam, The Netherlands, 1308 In: Editor (ed)^(eds) Book Efficacy and safety of anamorelin $\mathrm{HCl}$ in NSCLC patients: results from a randomized, double-blind, placebocontrolled, multicenter phase II study. European Cancer Congress. Amsterdam, The Netherlands, 1308 City

13. Temel J, Bondarde S, Jain M, Yan Y, Duus EM, Allen S, Mann W (2013) Evaluation of quality of life from a phase II study of anamorelin $\mathrm{HCl}$ in NSCLC patients. 2013 ASCO's Quality Care Symposium. In: Editor (ed)^(eds) Book Evaluation of quality of life from a phase II study of anamorelin $\mathrm{HCl}$ in NSCLC patients. 2013 ASCO's Quality Care Symposium, City

14. Abernethy AP, Temel JS, Currow D (2013) Phase III clinical trials with anamorelin $\mathrm{HCl}$, a novel oral treatment for NSCLC cachexia. In: Editor (ed)^(eds) Book Phase III clinical trials with anamorelin $\mathrm{HCl}$, a novel oral treatment for NSCLC cachexia, City

15. Grand S (1968) Color-word interference: an investigation of the role of vocal conflict and hunger in associative priming. J Exp Psychol 77: $31-40$

16. Cella DF, Tulsky DS, Gray G, Sarafian B, Linn E, Bonomi A, Silberman M, Yellen SB, Winicour P, Brannon J (1993) The Functional Assessment of Cancer Therapy scale: Development and validation of the general measure. Journal of Clinical Oncology 11:570-579

17. Smith E, Lai JS, Cella D (2010) Building a measure of fatigue: the functional assessment of chronic illness therapy fatigue scale. PM R 2:359-363

18. Ribaudo JM, Cella D, Hahn EA, Lloyd SR, Tchekmedyian NS, Von Roenn J, Leslie WT (2000) Re-validation and shortening of the Functional Assessment of Anorexia/Cachexia Therapy (FAACT) questionnaire. Qual Life Res 9:1137-1146

19. Cella D, Eton DT, Lai JS, Peterman AH, Merkel DE (2002) Combining anchor and distribution-based methods to derive minimal clinically important differences on the Functional Assessment of Cancer Therapy (FACT) anemia and fatigue scales. J Pain Symptom Manag 24:547-561

20. Eton D, Cella D, Yount S, Peterman A, Neuberg D, Sledge GW, Wood W (2004) A combination of distribution and anchor-based approaches determined minimally important differences (MIDs) for four endpoints ina breast cancer scale. J Clin Epidemiol 57:898-910

21. Yost KJ, Eton DT, Garcia SF, Cella D (2011) Minimally important differences were estimated for six patient-reported outcomes measurement information system-cancer scales in advanced-stage cancer patients. J Clin Epidemiol 64:507-516

22. Yellen SB, Cella DF, Webster K, Blendowski C, Kaplan E (1997) Measuring fatigue and other anemia-related symptoms with the Functional Assessment of Cancer Therapy (FACT) measurement system. J Pain Symptom Manag 13:63-74 